This item was submitted to Loughborough's Research Repository by the author.

Items in Figshare are protected by copyright, with all rights reserved, unless otherwise indicated.

\title{
Confidentiality in sports medicine
}

PLEASE CITE THE PUBLISHED VERSION

http://dx.doi.org/10.1016/j.csm.2015.10.006

PUBLISHER

(c) Elsevier

VERSION

AM (Accepted Manuscript)

PUBLISHER STATEMENT

This work is made available according to the conditions of the Creative Commons Attribution-NonCommercialNoDerivatives 4.0 International (CC BY-NC-ND 4.0) licence. Full details of this licence are available at: https://creativecommons.org/licenses/by-nc-nd/4.0/

\section{LICENCE}

CC BY-NC-ND 4.0

\section{REPOSITORY RECORD}

Malcolm, Dominic. 2019. "Confidentiality in Sports Medicine". figshare. https://hdl.handle.net/2134/19259. 
Title: Confidentiality in Sports Medicine

Author: Dominic Malcolm, PhD

Contact Address: School of Sport, Exercise and Health Sciences, Loughborough University, Loughborough, Leicestershire, UK, LE11 3TU

Tel: 441509226337

Fax: 441509226301

Disclosure Statement: The author has nothing to disclose.

Key Words: ethics, confidentiality, sports medicine, practice, policy recommendations

\section{Key Points:}

While ethical principles identify ideal practice, ethics are always operationalized in a social context and understanding that context is necessary for facilitating best practice.

Sports clinicians work in a context which, if not unique, is distinct from many other areas of medical practice.

The maintenance of confidentiality is shaped by the multiple obligations, physical environment, and practice and policy context of sports medicine.

Empirical research shows that there is considerable diversity of practice in relation to maintaining patient-confidentiality in sports medicine.

A variety of policy recommendations have been made that could enable greater conformity to medical ethical best practice.

\section{Synopsis}

This article synthesizes existing literature to provide a comprehensive summary of the ethical issues related to patient confidentiality in sport. It consists of four parts. The first outlines the medical ethical principle of confidentiality and identifies cross-cultural ethico-legal variations which shape its implementation. The second explores four factors specific to the context of sport which shape the application of patient confidentiality, namely: clinicians multiple obligations, physical environment and practice and policy context. The third reviews research detailing real life experiences of maintaining patient confidentiality in sport, and the fourth summarizes the many policy recommendations that have been made for enabling and enhancing compliance with this ethical principle. It is argued that the context of sport exacerbates pressures on clinicians to break patient confidentiality, breaches therefore occur relatively regularly, and interventions are required to enhance ethical compliance in sports medicine. 
It is often claimed that the ethical challenges of sports medicine are unique [1,2,3,4]. While claims to uniqueness may be overstated [5], the peculiarity of sports medicine is clearly illustrated by the challenges of maintaining patient confidentiality. Confidentiality has been identified as both one of the most important ethical issues in sports medicine [4,6], and empirically demonstrated to be amongst the ethical dilemmas most frequently encountered by sports doctors [7].

Initial analyses of confidentiality in sports medicine emerged due to a belief that the specific context of sport problematized compliance to ethical practice. However, these early works were overwhelmingly personal reflections, primarily related to individuals’ experiences and particular sports, and thus largely anecdotal [8]. In contrast, this article provides a more systematic and comprehensive understanding, through an overview and synthesis of existing literature. Specifically, it:

- outlines the medical ethical principle of confidentiality and identifies crosscultural ethico-legal variations;

- explores four factors specific to the context of sport which shape the application of patient confidentiality;

- reviews existing research which details practical experiences of maintaining patient confidentiality in sport; and

- $\quad$ summarizes the many policy recommendations that have been made for enabling and enhancing compliance with ethical practice.

\section{The principles of confidentiality and ethico-legal variations}


Historically the sovereignty of patient confidentiality has been central to medical ethics. It is the concluding item of the Hippocratic Oath. The importance of confidentiality stems from the notion that doctors should work wholly and exclusively on behalf of their patients and thus it is inextricably linked to privacy, patient autonomy and informed consent. However it is ethical to share information with other medical providers directly involved in the patient's healthcare where that information is pertinent to treatment [9]. Additionally five further mitigating circumstances that might necessitate non-consented disclosure can be identified: incapacity of a patient; medical emergency; legal obligation to state law (but not, it should be noted, sport-specific regulations and hence the physician has no ethical obligation to disclose athletes' use of performance-enhancing drugs); protecting the patient's health and wellbeing; protecting a third party from serious harm [10].

Despite this the literature has frequently identified the difficulties of simply transplanting medical ethical conventions into sports medicine [2]. While some explain this by locating sports medicine as a form of occupational medicine [11], it should be noted that even the Faculty of Occupational Medicine views patient confidentiality as a primary ethical issue [12], and that most ethical codes for sports medicine explicitly state that they add detail to, rather than replace, more widely recognized medical ethical principles $[13,14]$. The Code of Ethics published by the Fédération de Médecine du Sport (FIMS) explicitly states that “The physician’s duty to the athlete must be his/her first concern and contractual and other responsibilities are of secondary importance” [15].

Perceptions of ethical differences have led to a correlative tendency in the literature to highlight the importance of clearly distinguishing between what has variously been termed the primary general practitioner and contracted medical official [9], the personal and team physician [16], and the therapeutic and assessment roles of sports medicine [14]. For instance Bernstein et al. argue that the team physician is obliged to work in the interests of the (sports) 
organization which employs them, while the personal physician is unequivocally devoted to the individual patient (as an aside we might ask if any doctor always works solely in the interests of the patient uninfluenced, for instance, by budgetary constraints) [16]. However, being a team physician does not entirely dissipate all obligations to patient confidentiality. While the team physician is ethically obliged to prevent harm to other team members (e.g. informing them of another players' infectious disease) this would not necessarily extend to a more individual issue, such as an athletes' alcohol misuse [2]. The use of the term "physician covering the team" rather than "team physician" has been proposed to clarify the obligations the role should entail [4].

While these terminological differences are widely discussed, their implications are variously interpreted. The views expressed by British and Australasian authors and ethics codes are that the medical officer/team physician/assessment role is relatively discrete in practice. Conversely North American authors imply that this term encompasses almost all of the sports physician's practice and that because patients autonomously and voluntarily associate with teams, they thereby accept their rules regarding information disclosure [4]. It has been argued that as there can be no confidentiality in sports medicine, the team physician's only alternative is to make athletes aware of their obligation to share information with other medical, coaching and institutional personnel [17].

This difference can partly be explained with reference to the particular legal structure in the United States where physicians are bound by two specific laws [18]. According to the Health Insurance Portability and Accountability Act (HIPAA) an athlete’s medical records may be deemed to be part of their employment record rather than privileged health information and thus can be shared within (but not outside) the employing organization [6]. Secondly the Federal Education Rights and Privacy Act (FERPA) allows disclosure of medical information with those who have an "educational interest" (i.e. not with the media or 
third parties, but potentially with athletic trainers whose status is somewhat ambiguous in this respect) [2]. Because ethical codes operate within legal systems that are primarily statebounded, cross-cultural variations in the maintenance of confidentiality inevitably exist. Ultimately, "the idea of a universal code ... of sports medicine ethics is almost certainly a pipe dream” [5].

These cross-cultural differences illustrate a key sociological point about professions and their ethical codes. Ethical principles, although designed and claimed to be universal and immutable, operate in social contexts which are variable and dynamic. Sociologically considered medical ethics exist because of the social function they perform for the profession; namely bolstering the sense of trustworthiness and reliability which enable medicine to wield significant social power [19]. Where ethical princples hamper the influence of the medical profession, they will be modified or discarded. Thus, aside from legal differences, it is important to consider the sport-specific contextual factors which influence the way patient confidentiality is operated in practice.

\section{Contextual factors influencing confidentiality in sports medicine}

In identifying the following factors the aim is not to legitimize behavior which can be seen to compromise best practice, but to understand the social constraints on the operationalization of the ethical principles of patient confidentiality. Four main factors specific to the sports medicine context can be identified.

1) Multiple and conflicting obligations. The sports medicine physician is likely to experience multiple and conflicting obligations due to: a) sports organizations being the employers of medical personnel; b) the competitive aspirations of players overriding health concerns; and c) 
the cost of injury prevention relative to short and long term morbidity [6]. This has led the doctor-patient dyad to be reconceptualized in sport as the doctor-patient-team triad [2]. Conflicts of interest arise over the different priorities and values of the respective parties, and are complicated by the peculiar economic/contractual relations in sports medicine. For instance, doctors are frequently motivated by fandom [20] or ego and social status [1]. In some cases physicians pay significant amounts of money for the "privilege" of working with a professional sports team [2]. These factors contribute to the relative powerlessness of physicians within the sports context, manifest in the prominence of behaviors which serve to build trust and demonstrate usefulness [21]. Sports medicine has consequently been conceptualized as a "clients dependent practice” [22].

2) Physical environment. Because sports are particularly hazardous workplaces in which injury is common, it is inevitable that some medical care will be delivered in front of fellow athletes, coaches, etc. Such situations may be health-related emergencies, but others may 'only' be emergencies in the context of sports performance. This environment may therefore expose the clinician to well-meaning questions from concerned teammates [23] and stimulates management demands for prognoses. The openness of sports medicine treatment is compounded by the design of treatment facilities in sport which conventionally have little regard for athlete-patients’ privacy [7,24].

Moreover, sports medicine may be practiced on the playing field in front of thousands of spectators. This fuels a widespread (mis)conception that information about athletes' injuries is public knowledge. Orchard seemingly views the non-existence of privacy as an inconsequential "side effect of the enormous pay packet afforded to a professional football player" and is sanguine about the circumvention of patient confidentiality issues, arguing that as "part of the entertainment ... in this sense no one 'owns' the information” [25]. While this position is directly counter to (sports) medical ethical codes, it is clear that the accessibility of 
information about athletes' medical conditions creates a distinct environmental context which constrains clinicians' maintenance of patient confidentiality.

A final but increasingly important feature of the physical environment of sports medicine is the media. While much potentially sensitive medical information is benignly disseminated, journalists are also malignantly proactive in this process. In 1980 Peter Sperryn complained about the "constant pryings of the media” and information leaks from "hospital switchboards” [8], but the expansion of media outlets (e.g. the internet) has heightened this issue. Consequently medical information appears throughout the media and while there may be multiple sources for that information, the pressures on clinical staff to release confidential patient data are considerable [26]. In an age where professional reputations and commercial value are fostered via social media, the incentives to act unethically are increased [27].

3) Practice context. Sports medicine is fundamentally a multi-disciplinary practice. Ryan, for instance, has argued that sports medicine practitioners may include, “physicians, coaches, trainers, exercise physiologists, psychologists, sociologists, physical educators and others whose special interests are less well-defined” [28]. Clinicians therefore tend to work in multidisciplinary sport science teams (MSSTs) alongside colleagues whose degree of training, familiarity and concordance with medical ethical principles will vary. "With so many differences between disciplines, how is the athlete to distinguish who says what to whom?” [29].

The adversarial nature of sports medicine practice further complicates confidentiality issues. Elite sport entails continuous experimentation on the human body assessed through physical competition. Athletes are subject to on-going performance testing, from GPS tracking to the monitoring and assessment of supplement use. Medical staff are varyingly engaged in these performance-based activities; indeed the ACSM's Team Physician 
Consensus Statement argues that it is "essential” that the team physician embraces performance issues [18]. Healthcare personnel may even be included in performance-based financial reward systems [22]. Thus the medical and non-medical spheres in the practice environment of sport are frequently blurred. Further complicating confidentiality issues therefore, is the frequency with which individuals perform roles which span both healthcare and performance.

The complexities of sports medicine's practice context are illustrated by the system of transferring athletes between clubs, or from colleges to professional teams. This creates gray areas regarding confidentiality. Given the speed with which many transfers occur it is often impossible to get signed and informed consent from a player to release confidential medical information. Player movement raises the question of what information is owned by the sports organization (e.g. results of particular interventions) and what belongs to the player? Moreover, what is the duty of the club/college to disclose the medical condition of the asset they are releasing? [6]. Finally, consider the aborted transfer of Ruud van Nistelrooy in 2000 due to concerns revealed during the medical conducted by the purchasing club. The abandoned transfer became a global news story, but who released that information?

4) Policy context. Sports physicians work within the constraints of a variety of sports-specific policies which require the routine disclosure of otherwise privileged information. While we can assume that athletes largely consent to these disclosures, their choices are highly constrained by their desire to participate in sport. Policies relating to the screening and policing of sports participants are particularly problematic.

a) Testing for performance-enhancing drugs has been widely criticized for infringing the basic human right to privacy upon which the ethical principle of confidentiality is based. While such testing is frequently justified on the grounds that it prevents harm to individuals, 
safeguards equality, and is in the economic interests of sport, the most ethically challenging issues relate to:

- the "whereabouts system" which requires athletes to disclose their movements to enable no-notice, out-or-competition, testing;

- testing for recreational drugs such as marijuana which do not have performanceenhancing potential, but the use of which may be deemed to bring the sport into disrepute; and

- the therapeutic use exemption system where athletes must declare use of medications which may otherwise lead to positive tests (and thus potentially disclose medical conditions) [30].

The conflict between these drug-related policies and patient confidentiality is most clearly demonstrated in the 2009 World Anti-Doping Code. This stipulates that clinicians are themselves guilty of a doping offence if they do not report athletes' illegal drug use even when that action would be in contravention of their ethical duty to preserve patient confidentiality [31].

b) The procedures required to maintain sex-segregation in sport further entail the public disclosure of medical evaluations. The case of Caster Semenya showed that, based on little more than suspicion borne of visual impression, athletes could be subject to extensive medical and media scrutiny. While we do not know the detail of what investigations found, the outcome - that she qualifies to participate as a woman - is public knowledge. Similar issues relate to the criteria governing the participation of transsexuals in sport as outlined by the International Olympic Committee’s Stockholm Consensus. Sex segregation in sport necessarily entails the public dissemination of medical information that would otherwise be considered privileged and subject to patient-confidentiality [32]. 
c) Concerns have been expressed that cardiac screening, even when voluntary, may represent a "coercive offer" and so contravene the notion of informed consent [33]. However, policies which entail both mandatory testing and the enforced cessation of competitive sport (for some who test positive) are also problematic from a confidentiality point of view. While it may be argued that such policies are designed to protect individuals from harm, the resistance of some athletes who have been forced to withdraw from sport following such tests raises questions about the ethicality of this practice.

In conclusion, sports participation regulations frequently entail the public dissemination of privileged medical information (other examples include the pre- and postfight assessment of boxers, and the classification of athletes with disabilities). This policy environment is becoming more complex as diagnostic technology improves. For instance, concerns have been raised in relation to the potential of genetic testing which will require clarification regarding with whom data can be shared, and inevitably impact on the privacy of relatives [34]. In a context where such information is routinely shared, people are likely to become de-sensitized to the patient's right to confidentiality.

\section{Operationalizing patient confidentiality in sport}

A number of studies have examined how the medical profession, professions allied to medicine, and others who work in MSSTs operationalize confidentiality in sport. Three have focussed on doctors and physiotherapists in elite/professional sport, while a further five have concentrated on a wider range of healthcare professions, primarily in North American college sport. Comparison of healthcare in professional and college sport shows different emphases on the "culture of risk" and the "culture of precaution” which shape return to play decisions (often a key site for confidentiality conflicts) [35]. It has also been argued that athletes in 
individual sports are less open to the sharing of medical information than those in team sports [23]. These and other variations impact on how clinicians working in sport deal with patient confidentiality in their everyday practice.

Anderson and colleagues have explored the ethical issues concerning New Zealand sports doctors $[7,36]$. A half of respondents stated that they were contractually bound to disclose information to coaching staff and a third stated that they had disclosed information which athletes wished to remain confidential (examples related to infectious disease, the use of performance-enhancing and pain relieving medication, and pregnancy). They expressed concerns that: a) many coaches are unaware of the ethical constraints on clinicians to uphold patient confidentiality; b) sports organizations frequently do not have clear lines of communication; and thus c) controlling the flow of medical information is difficult. It was found that athletes were also often contractually-bound to waive their right to confidentiality [37].

The research revealed a high level of inconsistency amongst sports physicians. Most doctors stated that they would defy their contractual obligations and withhold information from coaches on the basis of the perceived higher ethical duty and practical importance of establishing patients’ trust. Alternatively to avoid conflict they might provide coaches with limited or partial information. Interviewees found the preservation of confidentiality in relation to performance-related information especially challenging [38].

Finally, this research also illuminated the media pressures related to confidentiality. Sports physicians described being continually pestered by journalists who had no regard for patient confidentiality. Experiences of media coercion included threats to fabricate stories if factual information was not provided [7]. Respondents sought to alleviate these pressures by, for example, drip-feeding information or repeating what was already known. However, they 
also revealed an awareness of the interdependence between injury reports, media exposure for a commercial franchise, the value of sponsorship rights and, ultimately, clinicians’ own economic interests [36].

Waddington and Roderick identified a similar lack of behavioral uniformity in research focused on clinicians working in English professional football [12]. Doctors and physiotherapists reported practice which ranged from strictly respecting patient privacy to an almost total disregard based on the view that it was essential and beneficial for managers to have full information access. Again differences were expressed about the differences when dealing with performance and non-performance related information (i.e. player injuries as opposed to social drug use). Physiotherapists, many of whom had only base level qualifications and, consequently, no experience of practicing in other healthcare settings, were perceived by doctors to be particularly culpable of breaking patient confidentiality. The authors concluded that medical confidentiality was poorly maintained in professional football because medical staff too often believed that a coach's status gave them the right to access all information.

Uniquely, this research also incorporated athletes’ views on confidentiality. Significantly, this showed that footballers routinely sought to minimize disclosure of information to medical staff due to the expectation that it would be divulged to management. Confidentiality breaches therefore had a negative impact on the quality of athletes' medical care.

Thirdly, Malcolm and Scott's research conducted with members of the British Olympic Association’s Medical Committee and Physiotherapy Forum found much more consistent recognition of the privileged nature of patient information [24]. These interviewees again reported that athletes' contracts frequently required them to waive medical confidentiality but 
noted that, when athletes resisted these obligations, clinicians were required to develop interpersonal strategies to minimize conflicts of interest. Strategies included:

- persuading athletes that disclosure was in their own interests (e.g. avoiding an unexplained decline in performance);

- suggesting that upholding patient confidentiality would lead the clinician to be bypassed by coaches leaving the athlete more exposed/isolated;

- highlighting how the athletes' actions could 'harm' others in the sports organization;

- co-opting clinical colleagues to exert pressure on the athlete; and

- “psychological tricks” to challenge the athlete’s rationale for non-disclosure.

In sum, and epitomizing the variability of practice, research with elite sport clinicians illustrates both strong paternalism and allegations of malpractice. Waddington and Roderick reported how a physiotherapist falsified information in order to protect a footballer with an alcohol problem [12], while Anderson [37] relayed clinicians' stories of creating fictitious physical injuries to obscure athletes' mental health issues. These reports mirror findings regarding clinicians’ avoidance of concussion diagnoses in order to obviate conflict with athletes and coaches (39). Conversely, in an alarming case of alleged malpractice, a footballer recalled a doctor threatening to release confidential medical information (which turned out to be false) in order to dissuade the player from seeking a lucrative transfer [12].

Five further studies have focussed on the attitudes and behaviors of practitioners incorporated in MSSTs

1) Sports psychologists. Reid et al.'s account of psychologists' experiences of working to establish an effective MSST focuses on the everyday challenges of confidentiality [40]. They identify differing communication protocols (including regarding confidentiality), driven by conflicts between the needs of individual athletes and the wider group, as a 
potential barrier to success. Their proposed solution rested on persuading athletes to permit full disclosure of personal data; in other words, effectively ignoring the rights of patients to confidentiality.

Complementing this study is Andersen's view that “horror stories” about confidentiality breaches amongst sports psychologists "are all too common” [41]. He notes that some disclosure may be linked to the constraints of certain national sports systems, legal requirements, or as a consequence of the conflicts of interest stemming from working with teams. However, significant harm is done to the profession by unlicensed, unchartered or unregistered "sports psychologists" who "routinely violate” the principles of confidentiality, including disclosing the names of celebrity clients to enhance their social and commercial status.

2) Athletic trainers. Research focussing on this group reveals confidentiality issues to be perceived as relatively infrequent [42], or as a recurrent, important, yet largely manageable [23]. Many athletic trainers reported that student-athletes were required to sign consent waivers but that any subsequent confidentiality conflicts that occurred could be dealt with by: a) challenging individuals to allow them to disclose; b) being vague or partial in information disclosure; c) contacting colleagues/mentors for advice; and d) seeking support elsewhere in the university. Student athletic therapists who, as peers, were more likely to be friends with the athletes they treated experienced particularly blurred practice boundaries. Yet the relatively controllable nature of these issues was attributed to the lesser economic pressures of the sport which, in turn, lessened pressures from and conflicts with the media. Additionally, athletic trainers' contractual arrangements with universities rather than individual teams, and the legal regulations related to FERPA, may also be significant. 
3) Fitness professionals. An Australian study revealed that this group received limited training in professional ethics which resulted, in part, in a variety of behaviors regarding confidentiality [43]. Specifically, large numbers (mistakenly) believed that sharing client data with colleagues and other gym members, and reporting suspicions of illegal substance use to criminal authorities were ethically acceptable practices.

Combined, empirical studies show that the reality of operationalizing confidentiality in sport is a wide variety of practice both between as well as within professions.

\section{Recommendations}

In light of the practical experiences of patient confidentiality in sports medicine, five categories of policy recommendations have been made.

1) Roles. Various authors recommend the clear separation of different sports medicine roles [3,5,38]. For those whose work is akin to occupational medicine (e.g. working as a team physician or in an assessment role) there is a clearer (but not unequivocal) case to be subject to full disclosure. Conversely those acting as general practitioners in therapeutic roles should maintain the highest ethical levels in relation to patient confidentiality. While a shortage of appropriately qualified and motivated personnel is acknowledged, ideally no individual would occupy multiple roles. To this end some recommend not only that players should have a primary physician outside of sport, but that sports organizations should encourage and enable athletes in this regard $[4,20]$. The broader legal framework will impact upon the appropriateness and feasibility of role separation.

2) Clarity. Building on the principle of informed consent, it has been noted that role separation requires that clinicians and athletes fully understand the healthcare system in 
which they operate [38]. For some this requires transparent reporting and information management systems [5]. Others have advocated the use of an "athlete’s charter" to identify which MSST personnel are bound by disclosure regulations [4,29]. A dialogue to establish what is deemed to constitute "serious harm" to third parties within sports teams is required as research identifies various interpretations of this notion [24]. Clarifying the appropriate balance of individual and group interests may alleviate pressures on disclosing confidential information. Robust ethical codes are essential to adding clarity [37].

3) Contracts. While written agreements contribute towards clarity of expectation, most researchers have warned against their use. For instance it has been argued that the contractual obligations of sports physicians should be limited to reduce the conflict between occupational expectations and medical ethical principles [37]. Similarly an athlete’s consent to disclose must be specific rather than general, the exception not the norm, and must be free of any element of coercion $[5,24]$. Indeed research shows that such contracts are redundant as both doctors and athletes consistently disregard their terms with seemingly limited sanction.

4) Education. In recognition of the variability in practice it is the duty of clinicians to ensure that they keep themselves updated on current ethical standards [4]. Calls have been made for sports organizations to develop and disseminate models of good practice [12], and enhance confidentiality-based continuous professional development [24]. Wider educational provision for sports psychologists [41] and coaching/training staff [4] has also been advocated which should be extended to all members of MSSTs.

5) Facilities. The provision of medical facilities which safeguard and/or maximize patient privacy has been recommended [24]. 


\section{Summary}

Ethics refers to idealized codes of behavior and consequently sports medicine ethics cannot be seen as unique or subject to lesser standards. It is however true that the social context in which sports medicine ethics are operationalized has distinct characteristics and manifestly structures the behavior of those charged with the duty to act ethically. Studies of the practice of maintaining patient confidentiality in sports medicine show, for example, that the distinct physical environment, practice and policy context of sports medicine, and the conflicts of interest which stem from multiple obligations shape how patient confidentiality is maintained. Breaches in confidentiality are relatively common and there is considerable variation within and between professional groups regarding the maintenance of patient confidentiality. There are however a variety of actions that could be taken to bolster ethical practice in sport in relation to patient-confidentiality and this implementation is vital to uphold the status and influence of sports medicine and the various related professions that populate MSSTs.

\section{References}

[1] Bernstein J, Perlis C, Bartolozzi AR. Ethics in sports medicine. Clin Orthop Relat Res. 2000;378:50-60.

[2] Dunn SR, George MS, Churchill L, et al. Ethics in sports medicine. Am J Sports Med 2007;35:840-4.

[3] Devitt BM, McCarthy C. “I am in blood Stepp'd in so far ...”: ethical dilemmas and the sports team doctor. Br J Sports Med 2010;44:175-78.

[4] Testoni D, Hornik C, Smith PB, et al. Sports medicine and ethics. The American Journal of Bioethics 2013;13(10):4-12.

[5] Holm S, McNamee M, Pigozzi F. Ethical practice and sports physician protection: a proposal. Br J Sports Med 2011;45:1170-3.

[6] Stovitz SD, Satin DJ. Professionalism and the ethics of the sideline physician. Curr Sports Med Reports 2006;5:12-124.

[7] Anderson L, Gerrard DF. Ethical issues concerning New Zealand sports doctors. J Med Ethics 2005;31:88-92. 
[8] Sperryn P Ethics in sports medicine - the sports physician. Br J Sports Med 1980;14(2/3):84-89.

[9] Faculty of Sport and Exercise Medicine. Professional Code. Edinburgh: FSEM; 2009.

[10] Exercise and Sports Science Australia. Code of professional conduct and ethical practice. Queensland, Aus: Exercise and Sports Science Australia, 2014.

[11] Crane J. Association football: the team doctor. In: Payne SDW, editor. Medicine, sport and the law. Oxford: Blackwell Scientific Publications; 1990:331-7.

[12] Wadddington I, Roderick M. Management of medical confidentiality in English professional football clubs: some ethical problems and issues. Br J Sports Med 2002;36:118-23.

[13] British Olympic Association. The British Olympic Association's position statement on athlete confidentiality. Br J Sports Med 2000;34:71-2.

[14] Australasian College of Sports Physicians' code of ethics and professional behaviour. New Zealand J Sports Med 2013;40(1):43.

[15] FIMS. Code of Ethics http://www.fims.org/files/8214/1933/5848/FIMSCodeOfEthics.pdf. Accessed 1 July 2015.

[16] Bernstein J, Perlis C, Bartolozzi AR. Normative ethics in sports medicine. Clin Orthop Relat Res. 2004;420:309-318.

[17] Johnson R. The unique ethics of sports medicine. Clin Sports Med 2004;23(2):175-182.

[18] Herring, SA, Kibler, WB, Putukian, M, Team physician consensus statement: 2013 update. Medicine and Science in sports and exercise 2013;45(8):1618-1622

[19] MacDonald, KM, The sociology of the professions. London: Sage; 1995.

[20] Wadddington I, Roderick M, Parker G. Managing injuries in professional football: A study of the roles of the club doctor and physiotherapist. Leicester: Centre for Research into Sport and Society; 1999.

[21] Malcolm D, Scott A. "Involved in every step”: How working practices shape the influence of Physiotherapists in elite sport. Qualitative Research in Sport, Exercise and Health 2014

[22] Malcolm D. Unprofessional practice? The power and status of sports physicians. Sociology of Sport Journal 2006;23(4):376-395.

[23] Riendeau C, Parent-Houle V. Legel-Gariel ME, et al. An investigation of how university sports team athletic therapists and physical therapists experience ethical issues. J Orthop and Sports Physical Therapy 2015;45(3):198-206.

[24] Malcolm D, Scott, A. Practical responses to confidentiality dilemmas in elite sport medicine, Br J Sports Med 2013

[25] Orchard J. Who owns the information? Br J Sports Med 2002;36:16-18.

[26] Ribbans B, Ribbans H, Nightingale C, et al. Sports medicine, confidentiality and the press. Br J Sports Med 2013;47:40-3. 
[27] Sports Physiotherapy New Zealand. Sports physiotherapy code of conduct, 2013. http://sportsphysiotherapy.org.nz/sportsphysiotherapy.org.nz/documents/SportsPhysiotherapy-Code-of-Conduct.pdf. Accessed 1 July 2015.

[28] Ryan AJ. Sports medicine in the world today. In: Ryan AJ, Allman FL editors. Sports medicine, $2^{\text {nd }}$ edition. San Diego: Academic Press; 1989, p.13.

[29] Collins D, Moore P, Mitchell D, et al. Role conflict and confidentiality in multidisciplinary athlete support programmes. Br J Sports Med 1999;33:208-11.

[30] Schneider AJ. Privacy, confidentiality and human rights in sport. Sport in Society 2004;7(3):438-456.

[31] McNamee M, Phillips N. Confidentiality, disclosure and doping in sports medicine. $\mathrm{Br}$ J Sports Med 2011;45:174-77.

[32] Caplan AL. Fairer sex: The ethics of determining gender for athletic ability. J Genet Counsel 2010;19:549-50.

[33] Anderson L, Exeter D, Bowyer L. Sudden cardiac death: mandatory exclusion of athletes at risk is a step too far, Br J Sports Med, 2012;46:331-334.

[34] McNamee MJ, Müller A, van Hilvoorde I, et al. Genetic testing and sports medicine ethics. Sports Med 2009;39(5):339-344.

[35] Safai P. Healing the body in the "culture of risk:” Examining the negotiations of treatment between sport medicine clinicians and injured athletes in Canadian intercollegiate sport. Sociology of Sport Journal 2003;20:127-46.

[36] Anderson L, Jackson S. Competing loyalties in sports medicine: Threats to medical professionalism in elite commercial sport. Int Rev Soc Sport 2012;48(2):238-256.

[37] Anderson L. Writing a new code of ethics for sports physicians: principles and challenges. Br J Sports Med 2009;43:1079-82.

[38] Anderson L. Contractual obligations and the sharing of confidential health information in sport. J Med Ethics 2008;34:e6.

[39] Malcolm D. Medical uncertainty and clinician-athlete relations: The management of concussion injuries in rugby union. Sociology of Sport Journal 2009;26(2):191-210.

[40] Reid C, Stewart E, Thorne G. Multidisciplinary sport science teams in elite sport. The Sport Psychologist 2004;18:204-217.

[41] Andersen M. "Yeah I work with Beckham”: Issues of confidentiality, privacy and privilege in sport psychology service delivery. Sp Ex Psyc Rev 2005;1(2):5-13.

[42] Swisher LL, Nyland J, Klossner D, et al. Ethical issues in athletic training: A foundational descriptive investigation. Athletic Therapy Today 2006;14(2):3-9.

[43] Dawson A, Andersen MB, Hemphill D The ethical beliefs and behaviours of Victorian fitness professionals. Journal of Science and Medicine in Sport 2001;4(3):266-282. 